\title{
Fresnel's Laws, ceteris paribus
}

\author{
Aaron Sidney Wright \\ aswright@stanford.edu \\ Forthcoming in Studies in History and Philosophy of Science
}

29 June 2017

\begin{abstract}
This article is about structural realism, historical continuity, laws of nature, and ceteris paribus clauses. Fresnel's Laws of optics support Structural Realism because they are a scientific structure that has survived theory change. However, the history of Fresnel's Laws which has been depicted in debates over realism since the 1980s is badly distorted. Specifically, claims that J. C. Maxwell or his followers believed in an ontologically-subsistent electromagnetic field, and gave up the aether, before Einstein's annus mirabilis in 1905 are indefensible. Related claims that Maxwell himself did not believe in a luminiferous aether are also indefensible. This paper corrects the record. In order to trace Fresnel's Laws across significant ontological changes, they must be followed past Einstein into modern physics and nonlinear optics. I develop the philosophical implications of a more accurate history, and analyze Fresnel's Laws' historical trajectory in terms of dynamic ceteris paribus clauses. Structuralists have not embraced ceteris paribus laws, but they continue to point to Fresnel's Laws to resist anti-realist arguments from theory change. Fresnel's Laws fit the standard definition of a ceteris paribus law as a law applicable only in particular circumstances. Realists who appeal to the historical continuity of Fresnel's Laws to combat anti-realists must incorporate ceteris paribus laws into their metaphysics.
\end{abstract}




\section{Introduction}

${ }^{1}$ If a science professor stops her philosopher colleague in the hall and they begin to discuss the scientist's picture of the world, what sort of objections could the philosopher make if they were unhappy with references to organisms and species, or to quantum fields and symmetry groups? If this philosopher has naturalistic leanings, there are three possible paths. Epistemological objections challenge scientists' access to the putative entities which they study. For example, Ian Hacking (1989) argued that astrophysicists' studies of black holes are so dependent on human assumptions and models that we should not accept that black holes have a mind independent existence. Social objections challenge the rationality or effectiveness of scientists' consensus formation. For example, the sociologist Harry Collins (1985) argued that experimental replications are question begging because the standard for a properly functioning instrument is its capacity to replicate the phenomena in question (Godin and Gingras 2002). Historical objections ask why a philosopher should believe their colleague now, when science's picture of the world has changed so often? What will the picture be next year? Larry Laudan (1981) formulated this challenge as a Pessimistic Metainduction (PMI; "meta" because he thought the underlying theories were inductive.) What is the best defence the scientist can make?

This paper is concerned with the historical challenge to believing in scientists' pictures of the world. It seeks a realist's response to a skeptical historicist. In order to specify the nature of this challenge, philosophers have appealed to a range of theories about science itself. Thomas Kuhn saw scientists as working within wordviews (weltanschaungen) which affected their research at the level of individual psychology, as well as at the level of communal values, ideologies, and institutions (Kuhn [1962] 1996, [1970] 1977). This picture is holistic and not well suited to formal analysis. Alternatively, formal analyses of science break it into pieces, such as sciences, fields, subfields, and individual theories, which are further divided into logical elements such as axioms, models of those axioms, and representation theorems which connect models to the world (Suppes 2002, ch. 1). In the tradition of Logical Positivism, they are less-concerned with metaphysical worldviews than with the structure of scientific reasoning, such as explanation. I will discuss Carl Hempel and Paul Oppenheim's paradigmatic "Studies in the Logic of Explanation" (1948) below. Elements from both the holistic worldview image of science and the formal image of science are at play in the debate over historical challenges to scientific realism.

Laudan's PMI is formulated in terms of a formal concept of scientific theories as consisting of sentences with theoretical terms such as "phlogiston," "aether," "caloric," "humour," etc. ${ }^{2}$ According to contemporary sciences, these terms no longer "refer"; that is, scientists no longer interpret these

1. I would like to thank anonymous referees, Peter Galison, David Kaiser, Chen-Pang Yeang, and especially Michael Friedman for reading and commenting on drafts of this paper. A poster version was presented at the 2016 Biennial Meeting of the Philosophy of Science Association in Atlanta; I thank those who joined in the discussion. This work was partly undertaken while I held a Postdoctoral Fellowship awarded by the Social Sciences and Humanities Research Council of Canada. All errors are my own.

2. In contrast, Paul Feyerabend's similar arguments were closer to Kuhn's worldview approach Feyerabend (1975) 2010. 
terms as referring to an entity in the world. Hilary Putnam put the argument in terms of terms:

just as no term used in the science of more than 50 (or whatever) years ago referred, so it will turn out that no term used now [...] refers.

Even if the empirical content of past theories is (approximately) included in newer theories, there is no guarantee that the terms in the old theory would have referents in the newer theory (Putnam $1975,184,180)$.

Putnam's solution to this problem appealed to both the formal- and worldview-images of science. He made concessions to the historicist argument, but sought to save scientific realism nonetheless. He gave up the possibility that terms like "ether" or "phlogiston" could refer. But he insisted that all the historical terms of past science were not alike-we continue to use some today:

it is a fact that we can assign a referent to "gravitational field" in Newtonian theory from the standpoint of Relativity theory (though not to "ether" or "phlogiston") [...].

Putnam argued that the acceptability of his limited realism about referents of theoretical terms depended on general epistemological commitments about knowledge and knowledge acquisition.

These retrospective reference assignments depend on a principle that has been called the "Principle of Charity" or the "Principle of Benefit of the Doubt"; but not on unreasonable "charity". Surely the "gene" discussed in molecular biology is the gene (or rather "factor") Mendel intended to talk about; it is certainly what he should have intended to talk about! (Putnam 1975, 180-81)

Here is where the holistic worldview image of science enters. The impact of general philosophical commitments on scientists' interpretation of evidence is a central feature of anti-realist argument. It questions the mind-independence of scientific reality. Could Einstein have convinced Newton to see gravity in a twentieth-century way? Did the two men's meta-scientific ideas about evidence and argument align? Putnam needed the answer to be "yes." Then he could say that the term "gravity" referred for both Newton and Einstein.

Against this thread of debate, John Worrall (1989b) made an influential argument for realism about scientific structures, such as laws of nature. He was not convinced that a realist could save the putative referents of scientific terms from the PMI, and he made his peace with what he thought survived across the centuries: the structure of the relations between the terms, regardless of what they stood for. In a philosophical sense Worrall took history quite seriously. He thought that the question of what survived theory change- the question of whether science in some sense progresses despite breaks—was "prior" to formal concerns about, say, approximate truth: "Unless this [pessimistic] picture of theory-change is shown to be inaccurate, then realism is surely untenable" (Worrall 1989b, 105, 109). Instead of focusing on the dustbin of the history of science (caloric, aether...), he focused on the monuments. Against pessimism, optimism (Worrall 1994). If theoretical terms did not survive scientific revolutions with their referring relations intact, philosophers should focus on what did survive (at least approximately). Newton's laws of motion and of gravity 
were developed in the Scientific Revolution and hold as approximations in current scientific theory. Worrall's strongest — and most influential—historical example came from nineteenth century optics and the proposal that light was a wave-phenomena in a medium, the luminiferous aether: Augustin Fresnel's Laws of the reflection and refraction of polarized light. According to Worrall, "continuity [across theoretical change] is one of form or structure, not of content" (Worrall 1989b, 117).

On one hand, Worrall gave up much more to historicists than Putnam did. Worrall gave up terms and reference. While Putnam tried to find a principled way to distinguish Newton's "gravity" from his "aether," Worrall was willing to allow neutrality about the entities which were attached to theoretical terms. Today, his proposal is called "Epistemic" Structural Realism because Worrall allows that there are ontologically-fundamental objects (or perhaps kinds, powers, etc.) beyond structures, but ESR denies that we have access to this level (Ladyman 1998). Whether "aether," "field," or "electron" feature in laws of nature, ESR sees limits to our ability to know whether theoretical terms truly refer. James Ladyman and Steven French have been the most prominent proponents of an "Ontic" Structural Realism, which put structures at the fundamental ontological level. In its most "eliminative" strain, they argue that structures are the only fundamental entities (Ladyman 2009). SR does not satisfy realists who desire a more straight-forward acceptance of the "face-value" of scientists' descriptions of the world (Putnam 1975, 193; Psillos calls this the realists' "semantic thesis" Psillos 2005, 385).

On the other hand, SR can present a much stronger challenge to antirealist arguments which are based on the worldview image of science. ${ }^{3}$ In SR, whether Fresnel believed in a material aether which was the medium of light propagation - whether he intended to study aethers, or action-ata-distance, etc.- - is irrelevant to the robustness of his laws throughout history. ${ }^{4}$ Putnam needed to borrow from the worldview image of science in order to combat a pessimistic reading of the history of science. Structuralists need not.

In order to maintain its distance from a holistic worldview image of science, SR needs to find a contemporary understanding of " scientific structure" which can be applied to past theories, and then show that such structures survived "radical” theory change, such as Kuhn's scientific revolutions. Structuralists do not need their notion of structure to be possibly-acceptable to long-dead scientists. As such, they do not need to rely on the sort of transhistorical philosophical commitments to which Putnam appealed. Posit for the moment that the historical case for continuity of structure can be made. The force of Laudan's PMI rests on a twentieth-century formulation of scientific theories as logical/linguistic entities. Any anti-realist who accepts a pessimistic induction

3. Over time, Worrall has framed SR as more or less dependent or independent on scientists' mindset. This is related to his view of the importance of novel predictions in the acceptance of scientific theories. See Brush 2007; Stanford 2003a.

4. It may be that Fresnel's commitment to the aether helped or hindered the reception of his views. My point here is that there is a historical fact of the matter that Fresnel's Laws were part of the "mature" science of optics from the 1820s, across the modern abandonment of the material aether, to today. Jed Buchwald's detailed study of this period places the emphasis away from abstract theories, and toward concepts and methods which were closer to experimental practice: Buchwald 1989, 1992. 
which is based on contemporary philosophy of science must also accept an optimistic induction which is based on contemporary philosophy of science (cf. Worrall 1994). This also applies to term-reference critiques which are not "inductions" (Feyerabend 1962; Ladyman and Ross 2007, 91-93). Even the most committed, holistic, worldview-based critique cannot contest that historical arguments are structured by the era in which they were written (Biagioli 1996; Iggers 2005). In this way, structural realism with a contemporary definition of structure offers a strong response to historicist critiques. ${ }^{5}$

If structures do survive, do they play the same role in contemporary science as in the past? Many realists are concerned about "fundamental" scientific entities. In a perceptive critique of contemporary scientific realism, P. Kyle Stanford uses an example from Francis Galton's population biology to show that a mathematical structure that has survived into modern population generics did not remain at the centre of the science of inheritance (Stanford 2003b, 571). Stanford is correct that scientists may change their assessment of a structure's fundamentality over time. But as long as the surviving structure is still a part of science-still explains or predicts or synthesizes empirical results; is still in the textbooks-there is no problem for realism here, per se. If on this basis a structural realist counts many laws as philosophically fundamental that a scientist would not, I am willing open this gap between philosophy of science and scientism. ${ }^{6}$

This paper is dedicated to a philosophical characterization of the structures which survive theory change, using the example of Fresnel's Laws (FL). The dominant characterization of laws and structure in the SR literature follows in the Logical Empiricist tradition of universal "exceptionless generalizations": true laws are true at all scales and at all locations at all times. The model is Newton's second law of motion: the force on a body equals its mass multiplied by its acceleration. ${ }^{7}$ FL are not like this. They refer to specific arrangements of material: beams of light, polarizations, and angles of reflection from plates of glass. I join Hempel and Oppenheim (1948), Earman, Roberts, and Smith (2002), and others in distinguishing between this sort of phenomenal law and more-abstract exceptionless generalizations.

Structuralists tend to either make an unstated inference that the survival of FL supports realism about all scientific structures, including universal exceptionless generalizations; or, they assimilate

5. Thus avoiding the trap of realists' "pyrrhic victories" over historicists, won at the cost of integrating historical attitudes into realism. Stanford 2003b; cf. Ladyman and Ross 2007, 91.

6. Considering the philosophical implications of historical change is certainly not a scientist's job. Against Ladyman and Ross's avowed scientism, Stanford sees the discovery of the metaphysics of the world as a joint project with scientists, historians, and philosophers. See Stanford in Stanford et al. 2010.

7. The "structures" and laws which are most commonly invoked in this literature are dynamical; they relate changes in the state of being of entities. And they are universal: any entity which counts as an object should follow Newton's laws. There are also less-universal generalizations. I.e. 'light travels at 299000 metres per second in a vacuum.' This is a ceteris paribus generalization because it refers to a context of applicability (a vacuum). 'Atomic oxygen is heavier than atomic hydrogen' is a true relation that holds without exception between two specific kinds of thing. It is not of the same level of generality of Newton's laws, or the universal law of gravitation. Nor does it describe a mutual interaction between bodies. Exceptionless generalizations of this most universal type are traditionally considered the 'fundamental' ones (Earman, Roberts, and Smith 2002). Specific-but-exceptionless generalizations may be closer to ceteris paribus laws, on my account. See section 4 . I thank an anonymous reviewer and Clark Glymour for prompting these comments. 
the survival of FL into an endorsement of the modern electrodynamics of Maxwell's Equations, and even quantum electrodynamics (Ladyman and Ross 2007, 284; French 2014, 154). I do not think this is an acceptable state of affairs. At its most conservative, SR should rest on the belief in the reality of just those structures which have actually survived theory change. With this narrower but stronger argument, I would hope to force an anti-realist or constructive empiricist to accept realism. However, this is rather beyond the scope of this paper.

Here, I propose to analyze FL as ceteris paribus laws, on the model of Nancy Cartwright (1983, 2002). The Laws hold, given certain physical conditions which are specified by ceteris paribus clauses. In sections 2-3, I follow the history of FL up to their present place in the field of nonlinear optics. FL are still used in physics, but their status has changed in two important ways. First, I argue that across the transition from aether-theories to modern-theories FL acquired new ceteris paribus clauses, and some of their ceteris paribus clauses became better specified. Second, I argue that the fundamentality of FL shifted. In the nineteenth-century, they were taken to bear on the fundamental ontology of light and the aether; currently, they are considered "phenomenological" relations which do not have fundamental, ontological, purchase (to physicists).

However my positive contribution to SR can only come after a correction to the reigningbadly distorted - image of the history of FL. Section (2) considers the claims of Worrall and Stathis Psillos about the history of optics and electrodynamics in the context the broad consensus of historians. Worrall presented FL as persisting over the radical ontological change from aether theories of light to modern theories, without an aether. And there is truth in that. The problem is that he claimed that light without an aether came about "long before the advent of relativity theory, when Maxwell's theory was accepted in its stead" (Worrall 1989b, 116). ${ }^{8}$ Ladyman (1998) approvingly cited this in his introduction of OSR, and the claim remains accepted in the literature (Ladyman and Ross 2007, 88; French 2014, 6). While it is true that many physicists, especially in German speaking countries, rejected Maxwellian aethers, they did not accept a field-propagation model; rather they opted for action-at-a-distance. But this was not what Worrall had in mind. Regardless, near the end of the nineteenth century, Heinrich Hertz's astounding demonstration that electromagnetic waves could be created in the laboratory caused a crisis for action-at-a-distance theories (Hunt 1991; Buchwald 1994). At the fin de siècle, Henrik Lorentz's aetherial electron theory was dominant (Hirosige 1969; McCormmach 1970). Historian Olivier Darrigol credits Emil Cohen with first elucidating our modern field concept, which was widely known only after 1910 (Darrigol 2000, 260-61, 366). Belief in a material aether-medium was not given up until well after 1905 and Albert Einstein's (1879-1955) interventions in kinetic theory, electrodynamics, and radiation theory (Einstein 1998; Schaffner 1972; Glick 1987). Well into the middle of the twentieth century, the aether concept was used in critiques of Einstein's theories, but it was also incorporated into them. In 1920, for example, Einstein ([1920] 2007) rejected the possibility that there existed

8. Henri Poincaré discussed the continuity of FL between changes in aether theories. See Connemara Doran, forthcoming, in Navarro 2018. 
a material aether - in the sense of a gas of molecules or an extended solid body — but he argued that the sense of an aether as signifying that "empty space" had physical properties was definitely included in the general theory of relativity. He proposed identifying the aether with the gravitational potential $\left(g_{\mu \nu}\right)$. Here, the word 'aether' is less important than the underlying physical concept. Maxwell and his followers may not have been sure about what sort of matter the aether was, but they were certain that it was some kind of matter. This material aether was rejected by Einstein, and eventually by the mainstream of modern science. ${ }^{9}$ In order for FL to have survived the radical ontological change from aether-theories to non-material-aether-theories, they must be followed into modern physics. (In the remainder of this paper, I will take 'aether' to mean 'material aether.')

In his historical claims, Psillos has returned to a ground of argument which Worrall sought to avoid: scientists' intentions. Implausibly, Psillos argues that Maxwell himself thought that one could study light and electromagnetism without a commitment to an underlying physical medium (Psillos 1995, 42-44; 1999, 131, 138). But because he sees the same central structuralist insight in Maxwell and in Worrall, Psillos insinuates Worrall's critical insight about structures to his image of Maxwell in a "Maxwellian-Worrallian stripe" (Psillos 2006, 567). This is important for Psillos's version of scientific realism because he relies on scientists to identify those parts of their theories which philosophers should withhold belief. He made this move as a defence against any charge that a realist simply cooked up an ad hoc criterion for identifying lasting bits of scientific theories in their challenge to the PMI (Psillos 1999, 111-13; Chakravartty 2007, 46; 2014; Stanford 2003b, 561). However, the scholarly consensus is that while Maxwell held complex and changing views about specific mechanical models of the aether, he maintained his commitment to the existence of a material aether (Everitt [1974] 2008; Morrison 1992; Hunt 2014). In section 2 I engage with Psillos's historical claims directly. Reception of Psillos's claim has been mixed. Marc Lange's review of Psillos's Scientific Realism (1999) is critical:

It is not the case (contrary to Psillos on p. 137) that Maxwell and his followers took the electromagnetic field as having physical reality independent of its embodiment in the aether. This came with Einstein (or perhaps Lorentz). (Lange 2001, 319)

Lange's review was not a full evaluation of these claims. Other reviews assert the opposite, for example that "[Psillos's] case studies from 19th century physics are authoritative and new" (Leplin 2000, 980). This paper corrects the record.

Proponents of SR have not embraced ceteris paribus laws (ie. Ladyman and Ross 2007, 256-57, 282-86). I argue that they should. In particular, OSR needs ceteris paribus laws in order to maintain the coherence of its sense of "structure." This is one of the most vexed questions for structuralists.

9. For recent studies on the range of attitudes and approaches to the aether concept after relativity, see the essays in Navarro (2018). I thank an anonymous referee for bringing Cordero (2012) to my attention. I agree with his challenges to those philosophers who would carve up scientific theories according to 'idle' vs 'explanatory' posits. And I appreciate his survey of attitudes to the aether in optics ca. 1900. However, it is a step too far to suggest that a particular stance on the aether was dominant throughout early twentieth century physics. 
As P. Kyle Stanford addressed it to Ladyman and Don Ross:

I doubt there is a single kind of structure that is simultaneously recommended to us by fundamental physical theorizing, preserved in the transitions between all or most suitably successful scientific theories, and sufficient to answer the realist demand to genuinely explain the success of our scientific theories. (Stanford et al. 2010, 164)

I agree with Ladyman and Ross's reply to Stanford that "this challenge can be met" (Stanford et al. 2010, 184). However, I argue it will require significant modifications to OSR at present. This is because proponents of OSR have excluded ceteris paribus laws from their definition of fundamental “structure." Ultimately, I would modify Stanford's desiderata, because what physicists label as "fundamental" is less important for realism than what philosophers choose to place under that label. Both the definition of "structure" and the definition of "fundamental" need to be independent of scientists' opinion if SR is to resist historicism.

A critical evaluation of Fresnel's Laws in philosophy of science has particular significance because of the singularity of the example within current debates. Contemporary "eliminitavist" strands of realism recognize the bite of the Pessimistic Metainduction and historical theory change, and shape their realisms around particular features of science that exhibit continuity over "revolutionary" breaks (Chakravartty 2014). For structure in particular, Fresnel's Laws are the primary historical example. Without the bright gleam of Fresnel's optics, structure can be no "weapon of the realist" (French 2006).

\subsection{Fresnel's Laws}

Before continuing, here is a formal expression of FL. For historical derivations see Fresnel ([1823a] 1866) and Buchwald (1989, 387-394); a contemporary reference is Born and Wolf (1999, \$1.5). In figure (1), the incident beam of polarized light shines down toward the boundary of two linear optical media at an angle $\theta_{i}$ to a perpendicular axis (cf. Fresnel [1819] 1866). The result is a reflected beam in Medium 1 with angle $\theta_{R}$ and a refracted (or transmitted) beam propagating in Medium 2 at angle $\theta_{T}$. Fresnel wanted expressions for the intensities of the reflected and refracted beams in terms of angles and indices of refraction. His picture of light as aetherial vibrations that were transverse to the direction of the beam allowed him to combine the physical optics of Snel's Law with conservation of des forces vives (slightly-anachronistically: conservation of energy and momentum). With one orientation of the polarization of light, he found that the intensity, $i_{R}$, of the reflected beam is given by:

$$
i_{R}=-\frac{\sin \left(\theta_{i}\right) \cos \left(\theta_{i}\right)-\sin \left(\theta_{T}\right) \cos \left(\theta_{T}\right)}{\sin \left(\theta_{i}\right) \cos \left(\theta_{i}\right)+\sin \left(\theta_{T}\right) \cos \left(\theta_{T}\right)}
$$

Similar results can be found for other orientations of the polarizability (cf. Worrall 1989b, 119).

In A Treatise on Electricity and Magnetism, Maxwell argued that his theory, with a unified elec- 


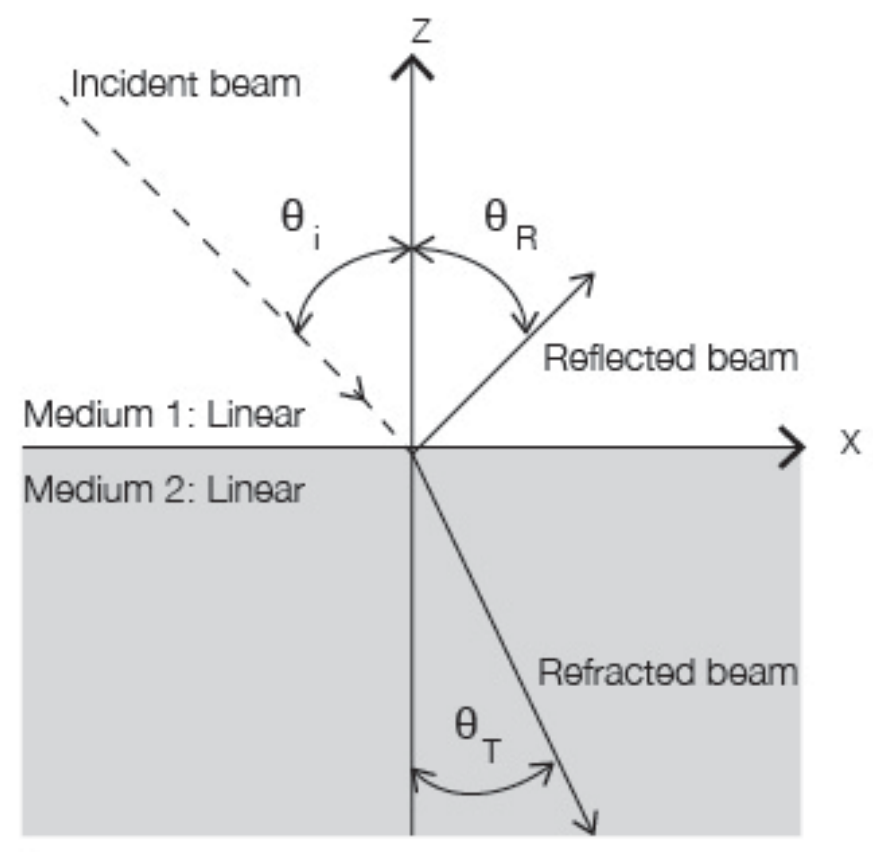

Figure 1: Fresnel's Incident, Reflected, and Refracted beams in linear media, after Chakravartty (2007, Fig. 2.1). Compare to Fresnel ([1819] 1866, 649). Credit: author.

tromagnetic and luminiferous aether, agreed with Fresnel's results (Maxwell [1891] 1954, \$797, 445). However, it was Hendrik Antoon Lorentz (1853-1928) who first derived Fresnel's Laws from Maxwell's theory in 1875 (Hirosige 1969, 160ff; Darrigol 1994, 268-70). In the group of physicists who developed Maxwell's theories after his death (Hunt 1991), Lorentz is accorded the most importance: According to Einstein, Lorentz achieved "the most important advance in the theory of electricity since Maxwell, by taking from ether its mechanical, and from matter its electromagnetic qualities." If Lorentz divorced the Maxwellian aether from mechanical qualities, Einstein placed his Special Theory of Relativity in Lorentz's lineage: "the whole change in the conception of the ether which the special theory of relativity brought about, consisted in taking away from the ether its last mechanical quality, namely, its immobility” (Einstein [1920] 2007, 615). Non-mechanical aether concepts—as labels for one or another entity in relativistic physics—are invoked to this day (i.e. Wilczek 2008; cf. Navarro 2018).

\section{Philosophy of science with history of science}

This section disputes two established claims about the history of nineteenth-century optics in the philosophy of science literature. They are:

W.1) Long before Einstein's relativity, the aether was rejected, and 
W.2) Maxwell's electromagnetic field was accepted in its place; and

P) Maxwell himself rejected the reality of the aether, or thought that the existence of

the aether was irrelevant to understanding optical and electromagnetic phenomena.

Aspects of each of these claims can be supported by the historical record. Many natural philosophers and men of science rejected the luminiferous aether, or various other subtle fluids associated with electricity, magnetism, and heat (W.1). However, they did not accept Maxwell's electromagnetic field in the twentieth-century sense (W.2). In fact, the fin de siècle was probably the high point of aether theory. At the time of Einstein's work in 1905, the dominant physical picture of light and electromagnetism was Lorentz's theory, which construed the aether quite minimally, but which was an aether-theory nevertheless (Lorentz 1916, 1). On claim (P): Maxwell famously developed a series of mechanical models of the aether over his career. And he reflected on the methodology of modelling natural phenomena, both in popular works and the details of his scientific writings. It has been the broad consensus of historians that throughout his work, Maxwell maintained an underlying belief in the physical reality of the aether (Everitt [1974] 2008, 213). In fact, Maxwell's great accomplishment can be characterized as unifying three subtle fluids into a universal aether for electricity, magnetism, and light (Schaffner 1972, 79; Siegel 1981). There is an extensive literature on nineteenth-century electromagnetism and optics which supports this analysis. ${ }^{10}$

On this basis, I conclude that the period from FL for Augustin Fresnel (ca. 1821) to FL in Maxwell's theory (derived by Lorentz in 1875) does not span a radical theory change in the sense of Kuhn's revolutions. It is only a change among aethers. Therefore, this span is an inappropriate frame for making arguments for scientific realism. The appropriate timespan for realist arguments based on FL extends into contemporary physics, which I sketch in section 3. (Those readers who are unconcerned with the historical details may jump to section 3 without losing the argument.)

\subsection{Pre-modern fields?}

In 1989 Worrall hoped to elucidate a proposal for scientific realism that captured "the best of both worlds" presented by realism and antirealism. Realism was supported by the "No Miracles Argument"; antirealism by an argument from history of scientific revolutions. Worrall was not concerned to formalize either side or even pin a picture to a specific author (Hilary Putnam, say). In broad strokes he sought to reconcile the general force of each argument by isolating one aspect of scientific theories that both survived theory change and made meaningful claims about the world that one could be a realist about. That aspect was "structure," which was picked-out by "the mathematical equations" (Worrall 1989b, 120).

Worrall rejected the suggestion that there is some way of seeing Fresnel as "really" referring to the modern electromagnetic field. Despite the personal influence of Imre Lakatos, "rational

reconstruction" had its limits. "Fresnel was obviously claiming that the light-carrying 'luminiferous

10. Influential works include: Buchwald 1985; Cantor and Hodge 1981; Darrigol 2000, chs. 4-5; Hirosige 1969; Hunt 1991, 2002, 2014; Jungnickel and McCormmach 1986; McCormmach 1970; Schaffner 1972; Siegel 1991. 
aether' is an elastic solid [...]. He was obviously claiming this, and it turned out that, if later science is right, Fresnel was wrong." Suggestions to the contrary-suggestions that Fresnel was "really" referring to a modern electromagnetic field—had "a definite air of desparation [sic] about" them (Worrall 1989b, 117). ${ }^{11}$

Worrall thought FL survived optical theory change (e.g. Worrall 1989b, 120). But he made a curious historical statement (with no citations) about which new theory instantiated this change. ${ }^{12}$ He wrote that Fresnel's aether was completely overthrown by later science, but that this occurred before Einstein:

Indeed this occurred, long before the advent of relativity theory, when Maxwell's theory was accepted in its stead. It is true that Maxwell himself continued to hold out the hope that his electromagnetic field would one day be 'reduced' to an underlying mechanical substratum-essentially the ether as Fresnel had conceived it. But in view of the failure of a whole series of attempts at such a 'reduction', the field was eventually accepted as a primitive entity. (Worrall 1989b, 116; compare Worrall 1994, esp. 340-41)

But it was not the case that the electromagnetic field was widely accepted as primitive before Einstein. ${ }^{13}$ And this discussion of 'reduction' is confused.

At no point between Maxwell's 1860s electrodynamics and Einstein's annus mirabilis in 1905 was there a consensus among physicists to abandon the aether and take up a self-subsistent electromagnetic field (an authoritative survey is Darrigol 2000). Hertz famously produced electric waves in his laboratory in 1888, confirming a prediction that Maxwell's followers had developed from electrodynamics (Buchwald 1994; Hunt 1991). ${ }^{14}$ That year, the Maxwellian George F. FitzGerald (1851-1901) gave a triumphal presidential address to the British Association for the Advancement of Science:

just as [Thomas] Young's and Fresnel's researches on the interference of light prove the undulatory theory of optics, so Hertz's experiment proves the ethereal theory of electro-magnetism. It is a splendid result. Henceforth I hope no learner will fail to be impressed with the theory-hypothesis no longer - that electromagnetic actions are due to a medium pervading all known space, and that it is the same medium as the one by which light is propagated; [...]. (FitzGerald [1888] 1902, 237)

The greatest triumph of Maxwell's theory was unquestionably seen as a triumph of an aether theory. This was the height of aether theory.

11. Others disagree: Psillos 1999, Ch. 12.

12. The issue here is the career of FL after their inventor's early death, at age 39, in 1827 . Worrall does accurately cite the primary source literature on Fresnel, particularly in his work on novel predictions. Worrall 1989a, 1994.

13. It may be possible to construct a more-abstract notion of "field" to draw out historical continuities, but that is not my concern here. Stein 1970.

14. On Hertz, John Mulligan (2001) has argued that he "retained his conviction of the aether's existence until his death on January 1, 1894" (157). 
True, British aether theories were not universally accepted in this period. Maxwell's Treatise was widely regarded as impenetrable, on both sides of the English Channel (Maxwell [1891] 1954; Warwick 2003, ch. 6). Those Continental European physicists who rejected the British aether also rejected British fields, and worked toward action-at-a-distance reformulations of Maxwell's theories: most prominently Wilhelm Weber and Hermann Helmholtz (Maxwell [1891] 1954, II, Ch. XXIII; Buchwald 1994; Hirosige 1969, 160-67). At the level of sub-disciplines: from the 1830 s to 1890 s, German optics research worked in a tradition of an elastic aether (Jungnickel and McCormmach 1986, II. 103-4). One exceptional individual was Cohen, a German Maxwellian, who judged the aether to be superfluous in 1900. Darrigol credits him with our contemporary grammar of "field" and "propagation" (Darrigol 2000, 260-61, 366). There were physicists who challenged Maxwell's theories and aether, but because they did not represent a consensus, and because they did not endorse a self-subsistent electromagnetic field, their views do not support Worrall's historical claims (W.1-2).

The electromagnetic field did gain significant independence in Lorentz's mature work. Tetu Hirosige argues that over Lorentz's career, he turned away from his two main influences-Maxwellian aether-dynamics and Weberian action-at-a-distance. In Lorentz's mature electron theory (18901928) Hirosige accepts Einstein's 1920 picture of Lorentz's work: that Lorentz removed all physicality from the aether, except its status as an absolute frame of reference (Hirosige 1969, 152; Einstein [1920] 2007; cf. Chalmers 2001, 435). This, Hirosige argues, made the electromagnetic field a self-subsistent entity (alongside the aether). For assessing Worrall's two-part claim, this may fulfill the requirement of an aether-independent electromagnetic field (W.2). Lorentz's work was more co-extant than "long before" Einstein's relativity; however, what is more important is that this independent field was co-extant with the aether. As such, Lorentz's work was not on the other side of a "revolutionary" or "radical" ontological break. And continuity on both sides of such a break is what realists need. As such, Hirosige's interpretation of Lorentz's work does not save Worrall's argument. (And while Lorentz's view was influential, diverse attitudes to the aether persisted.) In fact the aether persisted long after a Bern patent clerk declared it unnecessary; particularly at Cambridge, Maxwell's heirs gave relativity a rocky reception, at least through 1914 (Warwick 2003, Ch. 8, esp. 424-28).

Lorentz's work also shifted the electromagnetic field from fundamental status. ${ }^{15}$ Prior to this, the field represented the physical state of a fundamental entity in the world, the aether. Lorentz gave the field an independence from the aether. He was the paradigmatic aether theorist, who advocated an electromagnetic worldview, " $\mathrm{t}$ ]he simplest version of [which] held that electric particles are merely structures in the ether and that therefore the ether is the sole reality" (McCormmach 1970, 459). In retrospect, Lorentz's separation of the electromagnetic field and the aether was salutary. At the turn of the nineteenth century, the electromagnetic field had secondary ontological status: for Maxwellians, it was the state of motion of the aether, for Lorentz it was produced by singularities

15. I thank Michael Friedman for making this point. 
in the aether, such as the newly-discovered electron.

More than a question for theorists, the aether was also taken as physically real by experimentalists. Famously, Albert Michelson and Edward Morley (1887) performed experiments "On the Relative Motion of the Earth and the Luminiferous Ether." In fact, Michelson had read Maxwell's argument that Earth-bound experiments were too crude to measure the effect of the motion of the aether on light. Maxwell argued that astronomical measurements were the only option. Michelson took that as a challenge to his terrestrial-instrument-making skills (Everitt [1974] 2008, 215). Worrall's view of an aetherless turn of the century conflicts with our knowledge of experimental measurements of the effects of the aether, and their reception (Staley 2008). ${ }^{16}$ My point is simply that these turn-of-the-century physicists believed in an aether enough to try to measure its effects. If arguments (W.1-2) were correct, Michelson and Morley should have already rejected the aether and accepted a self-subsistent electromagnetic field; they would have felt no attraction to aetherial observations as a proving ground for their instruments. ${ }^{17}$

A possible source of confusion is revealed in Worrall's description of Maxwell's work in terms of an "electromagnetic field [which] would one day be 'reduced' to an underlying mechanical substratum" (Worrall 1989b, 116). Maxwell could not have hoped that the "electromagnetic field" would be "reduced" to an aether-for Maxwell, circa 1865, the physical "field" was space filled with aetherial matter. (The English sense of "field" here is of an area or region, as in "field of battle," "athletic field," etc. Compare to the French le champ or German das Feld.) This usage probably originated two decades earlier with Michael Faraday (Darrigol 2000, 98). In later usage, Maxwell considered the electromagnetic field to specify the state of the aether (see below). "For him [the field] was not an independent dynamical reality, but one of the mechanical states of a material substance" (Hirosige 1969, 156). Maxwell and his followers believed in matter and the aether, and employed a variety of models of it, and concepts which applied to it. According to Lorentz's 1906 lectures on electron theory at Columbia University, his audience knew

this theory of Maxwell, which we may call the general theory of the electromagnetic field, and in which we constantly have in view the state of the matter or the medium by which the field is occupied. (Lorentz 1916, 1)

Lorentz presented the electrodynamic field as a space which was "occupied" by aetherial matter; Maxwell's "field" theory was about the state of this matter in this space. Maxwell did not conceive of the electromagnetic field as an entity—as Worrall would have it—which could be reduced to further, more fundamental, entities. Over time, he considered the field to be a region, and then to be a characterization of the aetherial matter in a region. Neither of these could be "reduced" to a molecular or fluid aether (in the sense in which Maxwell worked to reduce thermodynamics to the kinetic theory of gases).

16. The aether drift experiments were unrelated to the origin of relativity. See Hirosige 1976; Holton 1988.

17. For later aether experiments see Schirrmacher and Lalli in Navarro 2018. 


\subsection{Maxwell against the aether?}

Worrall's picture of an aether-ambivalent Maxwell is common in the contemporary realism literature. A recent effort to "reconsider" the transition from Fresnel to Maxwell takes "Maxwell" from the current graduate course standard: J. D. Jackson's (1998) Classical Electrodynamics (Saatsi 2005). This anachronism was bolstered by Psillos's $(1995,1999)$ critique of structural realism and "standard" realist proposal. While there have been efforts to contest Psillos's depiction of past science, none has directly challenged his specific textual claims on Maxwell (Lange 2001; Chang 2003; Stanford 2003a).

It is necessary to correct the record. The main focus of my philosophical arguments is SR, not Psillos's "standard" realism. However, he has been a major interlocutor in debates over structuralism, and so a detailed discussion is warranted.

In his arguments, Psillos cites some source material from Maxwell, however I argue he misinterprets it. From Maxwell's 1878 Encyclopeadia Brittanica entry "Ether," Psillos concludes that

the upshot of Maxwell's point is that we can scientifically study and discover facts about the physical process of the propagation of light without being initially committed to any physical quantity as constituting light. (Psillos 1995, 43) 18

He argued that Maxwell shared Worrall's eliminativist insight, that ontological commitments to scientific work need not be holistic (Psillos 1995, 44; cf. Psillos 2006, 567).

Before specifying the nature of Psillos's error about Maxwell, here is the general tenor of the encyclopedia entry which Psillos cites, from its introduction and conclusion. While earlier aether theories had been imagined, Maxwell thought they failed because they

could not specify the nature of the motion of these media [...]. The only aether which has survived is that which was invented by [Christiaan] Huygens to explain the propagation of light. (Maxwell [1878] 1890, emphasis added, 764)

In concluding his discussion of whether the aether is molecular or was made up of vortices of a continuous fluid, Maxwell turned his attention skyward:

Whatever difficulties we may have in forming a consistent idea of the constitution of the aether, there can be no doubt that the interplanetary and interstellar spaces are not empty, but are occupied by a material substance or body. (Maxwell [1878] 1890, 775)

Maxwell's intervening discussion does not contradict his commitment to the aether. In fact, this sentence captures Maxwell's general view: though there were debates over the form of the aether, he had "no doubt" that one existed (Everitt [1974] 2008, 213). Maxwell wrote this Brittanica article at the height of his powers, as Cavendish Professor of Experimental Physics at Cambridge, while he was revising a second edition of $A$ Treatise on Electricity and Magnetism. (His death in 1879, at age 48, was due to cancer.) Here, I am making the most conservative form of historical argument.

18. Psillos misattributes Maxwell's entry to the publication date of Maxwell's 1890 Scientific Papers. 
Maxwell wrote an article on the aether, in which he wrote that he had no doubt about the existence of an aether-my argument is: believe him.

Returning to Psillos's treatment, he claims that Maxwell "distinguished between the geometrical character of the process of light-propagation and the nature of the physical quantity that constitutes light" (Psillos 1995, 42). This exhibits a similar confusion to Worrall's statements about "reduction" (sec. 2.1). Here is what Maxwell wrote about the constitution of light: he argued that light could not be a substance itself because interference patterns show that under certain circumstances light can destroy itself. "Now, we cannot suppose that two bodies when put together can annihilate each other; therefore light cannot be a substance" (Maxwell [1878] 1890, 764). In his text, Psillos follows Maxwell as he develops "vibratory" quantities that could mathematically characterize optical interference. However he skips Maxwell's definitions. "Such quantities are the measures, not of substances, but always of processes taking place in a substance. We therefore conclude that light is not a substance but a process going on in a substance" (Maxwell [1878] 1890, 764-5). This substance was the aether. Contra Psillos, Maxwell does not distinguish between a "process of lightpropagation" and a "physical quantity that constitutes light." For Maxwell, quantities did not constitute anything — they were measures—and light just was a process within the aether.

In his entry, Maxwell presented a number of possible forms of the aether, each with its own quantity. This quantity "may be [1] a displacement, [2] or a rotation, or [3] an electrical disturbance, or [4] indeed any physical quantity which is capable of assuming negative as well as positive values" (Maxwell [1878] 1890, 765-6). Psillos interprets this as indicating the independence of Maxwell's analysis from the underlying physical picture (Psillos 1995, 43-44; 1999, 113, 139). But it is important to distinguish the variety of models of the aether from the existence of the aether itself. In my view, this list of quantities should be read as a pedagogical statement that set up the remainder of Maxwell's entry. As it progressed, he introduced a series of experimental results and theories to further specify the relationship of light and aether. Fresnel believed in displacements of aetherial particles [1]; Maxwell had developed a theory of rotations of vortices in the 1860s [2]; Maxwell's unification of electricity, magnetism, and optics introduced electrical disturbances [3]; and Maxwell's mature work used very general specifications of material substances [4] (Lagrangian mechanics). The fact that Maxwell listed the various strands of aether-theory at the beginning of an encyclopedia entry should not be taken as evidence that Maxwell thought physical facts could be discovered apart from, or prior to, physical reality. But even if Psillos's reading of this sentence could be reasonably entertained, Maxwell was still referring to different processes within the aether. He should be read: light "may be a displacement [of the aether], or a rotation [of the aether], or an electrical disturbance [of the aether], or indeed any physical quantity [of the aether]." This reading is substantiated when Maxwell next "turn[ed his] attention to the medium in which it takes place. We may use the term aether to denote this medium, whatever it may be" (Maxwell [1878] 1890, 767). In different periods, Maxwell was agnostic about the correct model of the aether, but he did not express an atheism about the aether itself. Nor did he argue that the properties of the aether 
could be studied without believing in the existence of an aether.

The increasing generality over time of Maxwell's approach matched the generality of his mathematical methods: his Lagrangian mechanics accommodated multiple specific models of material, energy- and momentum-carrying aethers(cf. Stein 1989, 61-63; Psillos 1999, 139-40). In comparison to Newton's agnosticism (in print) over the cause of gravity-hypothesis non fingo-Maxwell was much more invested in underlying causes and entities. Daniel Siegel characterized Maxwell's new general mathematics as a "tactical retreat," in which Maxwell gave up the battle on a specific aether model in order to win the war for an aether against the action-at-a-distance theorists (Siegel 1981, 259). Alan Chalmers argues that Maxwell's tactics were imperfect, and that his Lagrangian methods failed to eliminate hidden mechanisms from electromagnetic theory (Chalmers 2001). According to Bruce Hunt, in this period "Maxwell still believed in a mechanical ether, but until more light could be shed on the details of its substructure, he thought it best to formulate his theory with a minimum of conjecture" (Hunt 2002, 318). Maxwell's method in the Treatise (first edition 1873) required only that the aether be a general material, capable of storing energy and propagating waves under energy conservation (Buchwald 1985, 20-40). These are general requirements for a substance. Contra Psillos, Maxwell's general mathematical methods were predicated on the existence of a substantial aetherial medium.

Psillos's second example of a self-subsistent electromagnetic field in his (1995) calls attention to Maxwell's 1864 argument before the Royal Society that "light itself is an electromagnetic disturbance in the form of waves propagated through the electromagnetic field according to electromagnetic laws" (the paper was published in 1865. Psillos 1995, 43). That is correct. But it only suggests an autonomous field when read with contemporary vocabulary and when read without the rest of Maxwell's text. "A Dynamical Theory of the Electromagnetic Field" was "called a Dynamical Theory, because it assumes that in that space [near electric and magnetic bodies] there is matter in motion.” Maxwell arrayed himself against Weber's action-at-a-distance theory, and argued for a "surrounding medium" that mediated electrical phenomena. Here is how Maxwell defined the electromagnetic field on the second page of his paper:

The electromagnetic field is that part of space which contains and surrounds bodies in electric or magnetic conditions.

It may be filled with any kind of matter, or we may endeavour to render it empty of all gross matter, as in the case of GeIssLen's tubes and other so-called vacua.

There is always, however, enough of matter left to receive and transmit the undulations of light and heat, and it is because the transmission of these radiations is not greatly altered when transparent bodies of measurable density are substituted for the so-called vacuum, that we are obliged to admit that the undulations are those of an aethereal substance, and not of the gross matter, the presence of which merely modifies in some way the motion of the aether.

We have therefore some reason to believe, from the phenomena of light and heat, that 
there is an aethereal medium filling space and permeating bodies, capable of being set in motion and of transmitting that motion from one part to another, and of communicating that motion to gross matter so as to heat it and affect it in various ways. (Maxwell 1865, 460)

In this paper Maxwell defined the electromagnetic field as a region of space filled with an aether. Waves travelling through the "electromagnetic field" were travelling through the aether-filled space surrounding charges and magnets. Recalling earlier generations of debates between vacuists and plenists (Grant 1981, Ch. 4; Shapin and Schaffer 1985), Maxwell rejected "so-called vacua," and supported the existence of the aether with the facts of heat and light transmission through glass Geissler tubes (see also Hirosige 1969, 154-55). Psillos claims that Fresnel's "luminiferous aether" and Maxwell's "electromagnetic field" referred to the same entity (Psillos 1999, 293-300). This is plausible because Maxwell's "field" referred to space filled with aether (ca. 1865) or the state of motion of the aether (ca. 1876). But Maxwell's "field" was not the same thing as our modern selfsubsistent electromagnetic "field." One simply cannot read our modern relativistic electrodynamics backward to Maxwell; in the mid-nineteenth century, the electromagnetic field was an aetherial region, not a self-subsistent entity.

Psillos also discusses Maxwell's optics in his Scientific Realism (Psillos 1999, 112-14, 130-40). Similarly to his analysis of Maxwell's "Ether" encyclopedia article (moderated somewhat on pp. 160-61), Psillos confuses cautious statements about specific models for rejection of any aether at all. ${ }^{19}$ In his 1861 paper "On Physical Lines of Force," Maxwell discussed his famous vortex and idle-wheel model of the aether (idle wheels are similar to ball bearings). As part of that discussion, Maxwell wrote: "I do not bring it forward as a mode of connexion existing in nature." According to Psillos, "it" referred to Maxwell's "model," which Psillos implies includes the famous vortices, the idle-wheels, and the aether itself (Psillos 1999, 138). But it is clear that this is not what Maxwell meant. He was not referring to the aether, nor to vortices. The "mode of connexion" was the "perfect rolling contact" without slippage that Maxwell assumed to hold between vortices and wheels (Siegel 1991, 41; Hunt 2002, 317-18; 2014, 306-8). Here is the preceding sentence: "The conception of a particle having its motion connected with that of a vortex by perfect rolling contact may appear somewhat awkward. I do not [...]" (Maxwell 1861a, 346).

It is appropriate to end with Maxwell's own words. At the conclusion of his Treatise, he considered the objections to his aether theory by action-at-a-distance theorists. Maxwell rejected mathematical entities such as Gauss's electrodynamic action and Neumann's immaterial potential. The fundamental fact was that light propagates and electrical and magnetic forces propagate. For Maxwell,

we are unable to conceive of propagation in time, except either as the flight of a material substance through space, or as the propagation of a condition of motion or stress in a

19. Psillos also claims the pre-Maxwellian work of George Green, James MacCullagh, and George Stokes as non-aetherial. All of these men proposed aether theories. See Schaffner 1972, esp. 59-68; Darrigol 2000, esp. 190-92. 
medium already existing in space.

Any attempt to mathematically describe this propagation must suppose there is some matter there. For if what propagates is Neumann's potential energy "how are we to conceive this energy as existing in a point of space, coinciding neither with the one particle nor the other?

In fact, whenever energy is transmitted from one body to another in time, there must be a medium or substance in which the energy exists after it leaves one body and before it reaches the other [...].

On the penultimate page, Maxwell admitted that Newton was correct to abjure the multiple aethers of the seventeenth century, "the properties of which were invented merely to 'save appearances' [...]." But with Maxwell's argument that his own electrodynamics and his Continental rivals' theories all "lead to the conception of a medium in which the propagation takes place," he concluded volume II:

and if we admit this medium as an hypothesis [contra Newton], I think it ought to occupy a prominent place in our investigations, and that we ought to endeavour to construct a mental representation of all the details of its action, and this has been my constant aim in this treatise. (Maxwell [1891] 1954, II: 492-93; cf. Maxwell 1861b, 162; cf. Everitt [1974] 2008, 213)

\subsection{Conclusion}

There are legitimate ambiguities in the corpus of Maxwell's statements about the aether, and the different threads of his views: on the existence of the aether; on the propriety of specific models of the aether; and on the limits of human understanding (Everitt [1974] 2008, 213). Philosophers have focused on Maxwell's use of models (Morrison 1992; Cat 2001; Chalmers 2001). In his longstanding Dictionary of Scientific Biography entry for Maxwell, the physicist and historian C. W. F. Everitt writes that

Maxwell's statements about the luminiferous ether have an ambiguity which needs double care in view of the intellectual confusion of much twentieth-century comment on the subject [...] the best short statement of Maxwell's position is that we may believe in the existence of the ether without direct knowledge of its properties. (Everitt [1974] 2008, 213)

This is roughly opposite to Psillos's position, which is that Maxwell inferred from his inability to precisely model the aether that the aether did not exist, or was irrelevant to electrodynamical research (Psillos 1995, 43). Hunt's authoritative overview of this period notes that Maxwell's unification of electricity, magnetism and light was a unification of aethers, not an elimination of all aethers (Hunt 2002, 317; Maxwell 1890, 500; also Siegel 1981, 254). Siegel has given a close 
reading of Maxwell's evolving electrodynamics that supports this dominant position among historians (Siegel 1991). Maxwell's drive toward unification was strongly connected to his devotion to evangelical Christianity; and his religiosity informed his view of the limits of human knowledge (Stanley 2012, 2015). Despite the complexities of Maxwell's life and work, it is the scholarly consensus that Maxwell maintained his belief in the aether throughout his career. This is supported by careful but plain readings of his published work.

With this historical record corrected, I now return to Structural Realism itself. Does this history affect a structural realist's philosophical conclusions about Fresnel's Laws? Yes. According to Worrall, in order to be a meaningful response to the challenge of major (or ontological, or revolutionary) theory change, the stability of FL needs to be seen across such a change (Worrall 1989b, esp. 115-17). The passage from Fresnel's elastic aether to Maxwell's vortex aether; to FitzGerald's "paddlewheel" or later "wheel and band" aether (1882-85); to Lodge's "cogwheel" aether (1888); or to Poynting's "turbine and spring" aether (1893) (Hunt 1991); or even to Lorentz's stationary aether, was much smoother and more incremental than the sudden revolutionary breaks that Kuhn saw as analogous to gestalt switches.

Of course if there was ever a theoretical break it came with Einstein's relativity. Could Worrall and contemporary structuralists simply push the timing of their analysis into the twentieth century? Don't we use FL today? Yes and yes. The problem for the realist is that in 1905 Einstein's three papers both ushered in a theoretical break in electrodynamics and set in motion the dethroning of FL from their position of ontological importance. Einstein's contributions to quantum theory combined with relativity to undermine FL's fundamentality, their ontological purchase (Einstein 1998).

\section{Today's view}

While Fresnel's Laws remain a standard part of the physical science curriculum, their stature is far from what it once was. The successes of Fresnel's physical optics no longer warrants belief in a luminiferous aether. Standard physical optics is seen as "phenomenological" physics that does not speak to fundamental questions at all. Part of the reason for this is atomism: the refractive index of bulk glass or quartz is seen as an average effect of more fundamental interactions. The larger reason is that FL and classical optics are empirically inadequate to describe the phenomena which manifests these atomic characteristics, such as the refraction of high-energy light (X-rays, etc.). Spurred by observations of intense laser light at the University of Michigan in 1961, the field of nonlinear optics has superseded classical (linear) optics as a candidate for understanding the interaction of light and matter (Bromberg 1991).

The fact that classical optical equations have limited applicability is not new to the philosophy of science literature. It was highlighted by Cartwright in a classic paper in 1980 (reprinted 1983), in the context of ceteris paribus laws. Cartwright used Snel's law as her motivating example, explaining 
that it only holds in linear media and quoting an optics text to the effect that in nonlinear media there will be two refracted beams (compare fig. 1) (Cartwright 1980, 160).

Fresnel's equations are built through combining the law of reflection, the conservation of energy, and Snel's law of refraction. Fresnel developed these equations and experimental apparatuses (with his friend François Arago) to study the properties of polarized light. He eventually proposed that the vibrations of the luminiferous aether that constituted light occurred only at right angles to the direction of transmission. This picture of aetherial molecules vibrating orthogonally to the path of a beam of light had empirical successes and ontological significance (Buchwald 1989). It was taken to support Huygen's hypothesis that light was a phenomena of vibrations in a luminiferous aether (against Newton's corpuscular hypothesis). Figure 1 is a diagram of Fresnel's process, made after Chakravartty (2007, Fig. 2.1).

In the first instance, FL are not universally applicable in the same manner as Newton's law of gravitation. There must be an interface between two media for light to reflect and refract. Not any medium will do. For example, if the medium is birefringent the refracted beam will depend on the orientation of the medium. Fresnel himself was steeped in experience with birefringent materials - before he invented his own polarizer (Fresnel's rhomb) he used them to generate polarized light in his own research (See Buchwald 1989, 223) and continued to use them as an object of study into the 1820s. Despite these conditions, FL were taken to bear on ontological questions in the nineteenth-century — as evidence for a molecular luminiferous aether. As Cartwright (1980) mentioned, for the equations of classical optics to hold, the materials under study must be linear (160). In the case of nonlinear media, and also in the case of very high-energy light, the departure from Fresnel's picture is dramatic (fig. 2).

A little formalism will elucidate the situation. In standard linear optics, the polarization of a medium, $P$, is related to the applied optical electric field, $E$, by the susceptibility $\chi^{(1)}$ :

$$
P=\chi^{(1)} E
$$

This can be generalized to arbitrary orders in a power series expansion:

$$
P=\chi^{(1)} E+\chi^{(2)} E^{2}+\chi^{(3)} E^{3}+\cdots
$$

in which the terms $\chi^{(2)}$ and $\chi^{(3)}$ are the nonlinear second- and third-order optical susceptibilities. These terms are directly related to a material's refractive index $n$ and give rise to qualitative and quantitative differences between linear and nonlinear optics. Some effects only manifest in materials with particular symmetry properties, but others — those related to $\chi^{(3)}$ —arise in normal materials such as glass (Boyd 2003, 2-3).

A nonlinear medium is characterized by an index of refraction $n=n_{0}+\delta n$, where $n_{0}$ is the normal contribution to $n$ and $\delta n$ is the non-linear contribution. In an analogous case to Fresnel's (and Chakravartty's) in figure (1): when the second medium is nonlinear, qualitative and quanti- 


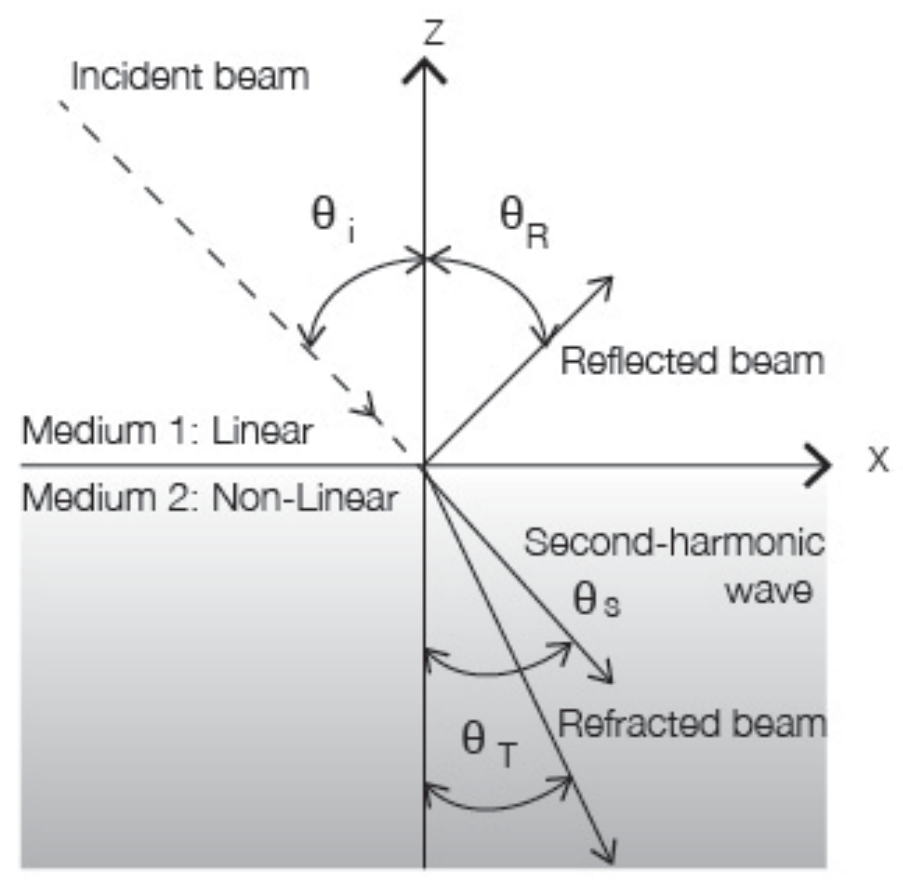

Figure 2: Nonlinear Optics at an Interface, after Boyd 2003, Fig. 2.11.2(a). Credit: author.

tative differences arise, depicted in Fig. (2). Most dramatically, the $\chi^{(2)}$ term has created a second beam of light (with double the frequency of the original beam), labelled "second-harmonic wave." For Fresnel, the index of refraction characterized the difference between the speeds of propagation of light in two media, and relied on Snel's Law (Fresnel [1823a] 1866, 757). In this case, because of the nonlinear term in the refractive index, $\delta n$, there is a nonlinear generalization of Snel's law (Boyd 2003, §2.11, 117-122).

But in fact we need not start off with comparatively exotic nonlinear media to observe these effects. In many materials the index of refraction is dependent on the intensity of the incident light, so that the nonlinear contribution to the refractive index is $\delta n=2 \bar{n}|E|^{2}$, with $\bar{n}$ a constant and $E$ the electric field strength (Boyd 2003, Ch. 4, "Intensity-Dependent Refractive Index," 189-235). For example, effects due to the $\chi^{(3)}$ term in $P$ (eq. 3) can be observed in air, water, ethanol, and many glasses (including $\mathrm{SiO}_{2}$, standard glass). This is to say that if the incident light is intense enough — whether a laser or highly focused sunlight—FL are inadequate, and no simple relations of approximation or correspondence obtain. For example, because beam splitting is qualitatively different from the phenomena FL describe, there is no simple sense in which the older relations are retained as a limiting case of the new. ${ }^{20}$ Formally, FL, eq. 1, relate the properties of three beams-

20. This change occurs regardless of the birefringence of the material, or lack thereof. As Landau and Lifshits put it, "small non-linear corrections to $\mathbf{D}(\mathbf{E})$ [the relation of electric field induction $\mathbf{D}$ and electric field intensity $\mathbf{E}$ ] cause qualitatively new effects" (Landau and Lifshits 1984, 372). 
incident, reflected, and transmitted. In order to be empirically adequate to describe beam-splitting phenomena, an entire set of new intensities and angles for the fourth beam would need to be added. Adding new variables to an equation is a qualitative change in the equation, a change of form.

Einstein's 1905 papers began the revolutionary theory change that structural realists need FL to survive. Einsteinian electrodynamics reformulated Maxwell's equations and made the luminiferous aether superfluous. The transition (Fresnel $\rightarrow$ Maxwell) was still (aether $\rightarrow$ aether), but (Fresnel $\rightarrow$ Einstein) gives (aether $\rightarrow$ field). At the same time, Einstein's atomism changed the status of FL: they could no longer be fundamental in a simple sense. Any reference to bulk properties of matter would now be considered secondary to a particulate analysis. The photoelectric effect compounded this non-fundamental status by connecting the interaction of light and matter to quantum theory. In addition to this changing fundamentality, FL were discovered to apply in more limited situations. But the brute specification of some ceteris paribus clauses have been made more-precise through appeals to modern theories of light and matter. I will now characterize this historical process as adding new ceteris paribus clauses to the Laws (relating to the types of media of propagation and the intensity of the incident light).

\section{Ceteris paribus}

Fresnel's Laws were part of a theory with fundamental importance in the 1820s, and they were a key aspect of successful novel predictions of the behaviour of polarized light (Worrall 1989a, 1994). They were not, however, universal "exceptionless generalization" laws in the sense of Hempel and Oppenheim (1948) or Earman, Roberts, and Smith (2002). FL applied to only particular beams of light in particular physical situations. In 1823, neither were they a specialization of such a universal "covering" law to specific circumstances (Nagel 1961). (Though, as discussed above, in 1875 Lorentz succeeded in deriving the Laws from Maxwellian electrodynamics and Lorentz's own theory of the aether within solid matter.) In this section, I will describe them as ceteris paribus laws, and situate them within recent debates on whether ceteris paribus laws are properly laws of nature at all. My purpose here is not to criticize the analysis of laws of nature in terms of "exceptionless generalizations" per se. Authors such as Hempel and Nagel in particular emphasized that they were studying the "logic of scientific explanation" and not necessarily scientific metaphysics. My concern is for a picture of laws of nature that is adequate to scientific realism as a "metaphysical hypothesis" (see Psillos 2005).

At present, there are two competing views on what makes a given expression a "ceteris paribus law." There is general agreement that they are not universal exceptionless generalizations, but there are disputes about less-abstract laws. For example, is an exceptionless generalization which has been specified to a particular physical application a "ceteris paribus law"? Or only a law with a theoretically-removable "proviso" (after Hempel 1988)? Earman, Roberts, and Smith (2002) characterize ceteris paribus laws as laws with specifications which cannot be removed, even in principle. 
Cartwright's response to this analysis is that she does not recognize the targets of her own work in those unruly, ineliminable, clauses (Cartwright 2002). In my analysis of FL, I find that they are ceteris paribus laws in the sense closer to Cartwright's. They do not have ineliminable ceteris paribus clauses. My aim here is not to confront SR with Cartwright's full ontological picture of a "dappled world" with causal powers or dispositions (Cartwright 1999). Rather, it is to confront SR with a different picture of structure. As such, I have chosen to focus on the trenchant and revealing analysis of the clauses themselves provided by Earman, Roberts, and Smith (2002) and Earman and Roberts (1999), which has been endorsed by structuralists (Ladyman and Ross 2007, with Spurrett, 283).

The Laws themselves (eq. 1), are equations with arguments corresponding to the angles, $\theta$, at which light approaches, reflects, and refracts (is transmitted) from the barrier between two isotropic (not birefringent) media; the index of refraction, $n$, of those media; the intensity, $i$, of the beams of light; and the plane of polarization of the incident light, $S$ (Fresnel [1823b] 1866). A schematic of this is presented in table (1). A direct connection to the aether is in Fresnel's interpretation of intensity, $i$, as the amplitude of the vibrations of molecules of aether. ${ }^{21}$ However, the structural realist should hope to cut the chain of scientific inference from this structure before reaching that now-discredited entity. SR has since become more nuanced, but "structure" has been parsed simply as the referent of "the mathematical equations" (Worrall 1989b, 120). I am widening that prescription to include ceteris paribus clauses. In parallel to Cartwright's (1980) discussion of Snel's law-in view of table (1) - I characterize FL as laws that only hold ceteris paribus: they hold so long as the conditions obtain. When considering the expression of FL, we should include not only the formula, eq. 1, but also include these ceteris paribus clauses.

$\left.\begin{array}{c|c|c}\text { Relations } & \text { Arguments } & \text { Conditions } \\ \hline \text { Fresnel's equations } & \theta, n, i, S & \begin{array}{c}\text { flat boundary between media } \\ \text { media are isotropic } \\ \text { light is polarized } \\ \text { media are linear } \\ \text { intensity not too high }\end{array}\end{array}\right\}$ ca.1820

Table 1: Table depicting the elements necessary for Fresnel's Laws to have physical sense and accuracy. (This already assumes that FL can be meaningfully isolated from the broader contexts of Fresnel's wave theory or Maxwellian's electrodynamics.)

21. I read Fresnel's molécules éthérées as a specific aether model, as compared to a continuous fluid or solid (cf. Psillos 1999, 158). 


\subsection{Legal distinctions}

Earman and Roberts (1999) think that the only true laws of nature are exceptionless generalizations of fundamental physics. They formulate these generalizations as counterfactuals, in idealized situations: were modern relativistic electrodynamics to be true, in flat spacetime, then a true law would be: "that the curl of the E field is proportional to the partial time derivative of the B field, etc.," one of Maxwell's Equations (446). Now, derivatives of fields are mathematical entities that do not stand in a simple relation to observable quantities (cf. Feyerabend 1962, 28 fn. 1). Earman and Roberts accept that exceptionless (or proviso free, in Hempel's (1988) terms) laws are tested through specific physical models. But they argue that physicists intend to test exceptionless laws when they use specific models; and that establishing exceptionless laws is the goal of physicists throughout history (Earman and Roberts 1999, 445-46). Because of this intention, genuine laws of nature must be exceptionless.

Earman and Roberts's appeal to the intentions of historical figures is parallel to Psillos's and Putnam's (sec. 1). I am less willing to give physicists' intentions philosophical weight-Maxwell intended his models to refer to the aether, and realists need to reject its existence. These appeals to intentions make trouble for realism. I also doubt the broad correctness of Earman and Roberts's claim that perfect generality was scientists' goal. There certainly were some, as Earman and Roberts cite. However, there are also counter-examples. The danger here is projecting a particular philosophical view of laws of nature-as universal and exceptionless-onto historical figures. Newton's Laws have been qualified since their introduction (cf. Earman, Roberts, and Smith 2002, 283-86). Here is the first of Newton's "Axioms, or the Laws of Motion" from the Principia Mathematica:

Every body perseveres in its state of being at rest or of moving uniformly straight forward, except insofar as it is compelled to change its state by forces impressed.

Projectiles persevere in their motions, except insofar as they are retarded by the resistance of the air and are impelled downward by the force of gravity. A spinning hoop [...]. (Newton [1687] 1999, 416)

If the statement of a law contains the word "except," there is little hope for it to be exceptionless. A modern counter-example is Paul Dirac, who believed that all scientific theories are approximations (Wright 2016).

The dominant view of laws in the philosophy of science is that they are universal and exceptionless. This holds over a wide range of further metaphysical views about laws. For example, on an empiricist view of laws - descended from Ramsey, and Lewis - a given law is nothing but a label for regularities in the world; Earman has endorsed this view. In contrast, a realist view of lawsexpressed by Dretsky, Tooley, and Armstrong — takes it that laws exist as part of the furniture of the world. Both empiricist and realist metaphysicians can agree that laws are universal and exceptionless. ${ }^{22}$ However, it is evident that this philosophical idealization is inapt for characterizing laws of

22. See Mumford 2005, and references therein. Earman: 401; regularity, universality, etc.: 404. 
nature as they were expressed in the history and practice of science (Cartwright 1980). ${ }^{23}$ In Newton's actual words, his laws are much less "liars" than on a philosopher's logical re-formulation, such as 'all Fs are Gs' (after Cartwright 1983). Instead, I think that ceteris paribus laws offer a better framework for discussing laws of nature. Here, too, multiple underlying metaphysical views are possible; for example, Cartwright's (1999) causal powers and SR (or so I argue).

What about the specific ceteris paribus clauses in FL? Earman, Roberts, and Smith (2002) would label some of the conditions in table (1) as simple "conditions of applicability" that are left unstated by "lazy" scientists. Laws concerning the refraction of beams of light are understood to need beams and media. While such conditions would preclude FL from being fundamental laws, they would still be scientific laws in good standing. Other conditions are more problematic. Here is the heart of the matter: Earman, Roberts, and Smith (2002) argue that the only ceteris paribus clauses that should worry philosophers are those that are "ineliminable" from the laws (283-84). Problems arise if we can never close-off the condition in a simple formulation. The argument against counting ceteris paribus laws as laws of nature is that expressions with these "ineliminable" clauses are no laws at all. They are not even well formed formal or linguistic objects. On the other hand, if all we have to do is require polarized beams and flat boundaries, there is no cause for concern. Advocates of the exceptionless generalization view can accept that laws with "conditions of applicability" are laws (even if not "fundamental"). I happily accept that "conditions of applicability" do not pose a problem for accepting FL as laws of nature.

Philosophers such as Ronald Giere (1999) and Marc Lange (2000) have argued that there are laws with ineliminable ceteris paribus clauses. Earman, Roberts, and Smith $(2002,284)$ complain that these philosophers construe ceteris paribus conditions such as 'requires beams of light' as "indefinite" and ineliminable only because they refuse to use the language of physics within their ceteris paribus clause. They have a good point. For the case of FL and intensities I labeled the condition on intensity in table (1) as "not too high." Modern physicists would specify this much more precisely: FL hold so long as the intensity of incident light—understood in terms of Einstein-Maxwell electrodynamics - does not approach the binding energy of electrons in the crystal lattice, or other relevant quantum mechanical features of the medium (Boyd 2003, Ch.s 3 and 5). For Earman, Roberts, and Smith (2002), specifying the terms of ceteris paribus clauses does not elevate a ceteris paribus law to a fundamental law. But the elimination of "ineliminable" ceteris paribus clauses does bring these relations within their overall framework of lawful explanations.

Are any of the conditions in FL ineliminable? On one reading, yes. There is no simple list of birefringent materials, and isotropic materials can become birefringent in different circumstances; for example in an electric field (the Kerr effect, discovered 1875). One can go some way to ordering the possibilities by referring to the 230 groups of crystal symmetries, but the number of possible crystals that can be formed may be limitless (Hahn 2006). The periodic table is not closed. Similar concerns exist with more general forms of non-linear media. And as discussed above, even isotropic

23. For this history before Newton, see Ruby 1986. 
and linear materials will not follow FL if the intensity of the light is high enough. Realists may have to accept that brute ceteris paribus clauses must be included in their fundamental structures.

However, it is possible to "close off" these ceteris paribus clauses. Take the example of the closure of the periodic table. It is the case that our collection of known elements is not a necessarily complete list. It is also the case that as elements fill-out the table they become less stable. We do not know why this is the case. The half-life of Tennessine (atomic number 117, named by the International Union of Pure and Applied Chemistry in 2016) is 0.051 seconds. This is clearly too short a lifetime in which to form crystals which could plausibly be tested in an optics experiment. In this way, an ineliminable ceteris paribus clause can be reconstituted as an eliminable quantitative limit on the atoms which make up stable matter (a minimum half-life) of the material conditions necessary for the Laws to hold.

With this manoeuvre, I view FL as neither exceptionless generalizations, nor among the dangerous, anti-unity-of-science ceteris paribus laws Earman, Roberts, and Smith (2002) are most concerned about. My view fits Cartwright's, who did not recognize the targets of her investigations in Earman, Roberts, and Smith's analysis (Cartwright 2002). FL only hold ceteris paribus ; and these clauses specify the physical situations in which the laws apply. These clauses may refer in a quantitative way to other physical theories, such as specifying the maximum intensity of beams of light in terms of the quantum theory of crystal lattices. Or these clauses may fall-short of this level of specificity and integration with other laws. Ineliminable ceteris paribus clauses make laws uncontainable in principle. Ineliminable ceteris paribus clauses cannot be specified in terms of other laws, and therefore threaten the unity of science and the primacy of fundamental physics over the special sciences (Earman, Roberts, and Smith 2002). But I do not see ineliminable ceteris paribus clauses applying here. FL can be defined in a finite set of words and symbols. And they can be connected to other scientific theories. In my view, this makes FL definite-enough structures to sustain realist commitment to their place among the constituents of the world.

\subsection{Legal dynamics}

I see the core relations of $\mathrm{FL}$ as continuous over time, but with changing ceteris paribus clauses. Some clauses changed, in the sense of becoming-specified to other theories, and some new clauses were added. Through this the central description of light reflecting and refracting at media remained constant. (Even the mathematical notation was quite stable.) Ceteris paribus laws with stable central expressions and dynamic clauses provide a framework for capturing what is conserved throughout scientific change (cf. Post 1971).

A historical dynamics of laws was an impossibility for Hempel and Oppenheim. They argued that laws are strictly true statements, with no allowance for laws which are true only relative to available evidence.

Thus, for example, we would not say that Bode's general formula for the distance of the planets from the sun was a law relatively to the astronomical evidence available in 
the 1770s, when Bode propounded it, and that it ceased to be a law after the discovery of Neptune and the determination of its distance from the sun; rather, we would say that the limited original evidence had given a high probability to the assumption that the formula was a law, whereas more recent additional information reduced that probability so much as to make it practically certain that Bode's formula is not generally true, and hence not a law. (Hempel and Oppenheim 1948, 152)

On my view, Bode's law was a law ca. 1770, and is still a law today, even if the addition of ceteris paribus clauses makes it rather unhelpful for understanding our solar system.

Hempel and Oppenheim were left with a kind of transcendental philosophy of laws of nature, in which " $t$ ] he requirement of truth for laws has the consequence that a given empirical statement $S$ can never be definitely known to be a law" (Hempel and Oppenheim 1948, 152). These laws are of central importance, but we can never know them. This connection between truth and general laws has carried through in the contemporary literature: "Typical theories from fundamental physics are such that if they were true, there would be precise proviso-free laws" (Earman and Roberts 1999, 446; cited in Ladyman and Ross 2007, 283). Hempel and Oppenheim were explicit that they required their picture of laws of nature in scientific explanation to fit with a particular theory of truth. In contrast, realist replies to historicist critiques are most concerned with the continuity of scientific reference.

Reliance on these in-principle-unknowable laws causes problems for SR. First a historical problem: If a structuralist rejects ceteris paribus laws, they must abandon their best historical example of structural continuity over revolutionary theory change. ${ }^{24}$ A logical procedure would be to look for universal exceptionless generalizations which have survived theory change. Unfortunately, (true) universal exceptionless generalizations cannot be identified. (They function more like regulative ideals, or "ideal knowledge achievement[s]" (Ladyman and Ross 2007, 286; cf. Earman and Roberts 1999, 446).) As such, an appeal to exceptionless generalizations to defeat the Pessimistic Metainduction is excluded. The first problem for structuralists is not whether scientists have epistemic access to exceptionless generalizations. The problem is whether philosophers and historians have access to true exceptionless generalizations to mount a defence against the PMI. I am not contesting that the search for exceptionless generalizations has motivated some scientists. I am arguing that whether-or-not that has been the case, exceptionless generalizations are an unsuitable basis upon which to build SR.

This is related to a contemporary problem for exceptionless-SR: there are no current structures in which to believe. Any currently-available scientific laws which are interpreted as "exceptionless"

24. It does not follow that once FL were derived from Maxwell's theory, the survival of FL somehow transfers to Maxwell's equations, quantum electrodynamics, and gauge symmetry groups, as French seems to suggest (French 2014, 154). This is particularly the case if proponents of OSR make a qualitative distinction between FL as ceteris paribus laws and Maxwell's equations as candidate-universal exceptionless generalizations. It is true that Maxwell's equations also survived the transition to modern physics. But they are now viewed as only accurate under certain physical limits, and are no longer viewed as fundamental. 
are false. ${ }^{25}$ As a realist, I want more than this. Like Cartwright, I want a realism that can ground human action (Cartwright 1999, Introduction). To take a non-optical example: the Earth's climate in an unobservable scientific entity. Our understanding of the climate has widely varied over time, and is now mediated through complex modelling practices (Edwards 2010). Nevertheless, the key realist argument which supports human action is the reality of the relation between increasing carbon dioxide levels and climate change effects. I see ceteris paribus laws as a framework for a philosophical understanding of contemporary, identifiable, structures which can undergird philosophers' commitment to their reality today.

My positive framing carries through the historical trajectory of ceteris paribus clauses. This has been discussed in the context of Ernst Nagel's logic of scientific reduction as a type of explanation (Nagel 1961, 1970; Sarkar 2015). Clark Glymour (1970) saw inter-theoretic explanations as more complicated than Nagel's logical deductions. For him, "one of the functions of inter-theoretical explanation is removal of ceteris paribus clauses which experience makes us impose on our laws and theories" (342). (For Glymour, "removing" a ceteris paribus clause is what I have referred to as "specifying" one with respect to another theory.) This fits well with my picture of the dynamics of ceteris paribus clauses. For a fundamentalist, the discovery of quantum behaviour is a blow. Einstein's Equations - considered as exceptionless generalizations, meant to be valid at all lengthscales_are falsified (as some "fundamentalists" accept Hoefer 2008, 319). For me, the discovery of ceteris paribus clauses - and their specification with respect to other theories-are clear advances of scientific knowledge. When we better know the limits of our equations, we better know the world.

\section{Conclusion}

In this paper I have considered historical and philosophical perspectives on Fresnel's Laws of optics. Philosophically, I characterize FL as ceteris paribus laws, understood on Cartwright's view. The content of FL is not only a series of mathematical expressions and linguistic terms, the content also includes the conditions under which the Laws apply. This is in contrast to another view of laws, in which laws do not include ceteris paribus clauses, and each law-statement is seen as a potential true, universal, and exceptionless generalization. There are, I think, two central complaints against the ceteris paribus picture. First, as Earman and co-workers have elaborated, if a given ceteris paribus clause is_-in principle_-ineliminable from a putative law-statement, this statement is too ill-defined to be counted as a law. In addition, these ineliminable clauses pose a barrier to quantitative pictures of the unity of science (in which all laws may be subsumed under a most-general law, or as mathematical approximations to a most-fundamental law, etc.). However, Cartwright

25. This is so, not because an exceptionless generalization needs to explain literally everything, but because structures like Maxwell's equations are not empirically adequate in all cases. Hence relativity, quantum theory, etc. It was clearly not Hempel's or Earman et al.'s intent, but a similar objection may be applied to analyses of scientific explanation in terms of exceptionless generalizations. Strictly speaking, on this view, has there ever been a scientific explanation? 
(2002) does not accept that ineliminable clauses are essential to the ceteris paribus picture of laws, and I agree. The second complaint to the ceteris paribus picture is that universal and exceptionless laws are properly 'fundamental' but ceteris paribus laws are not. Briefly, my view is the following: FL count as ceteris paribus laws; on the universal and exceptionless view, FL are either not laws or are not 'fundamental' laws. Insofar as realists appeal to the history of FL to rebut the Pessimistic Metainduction, they need a picture of laws which includes FL. Secondly, if a realist's response to the PMI is to be consistent with their specific metaphysical proposal (ESR, OSR, dispositions, etc.) they should use the same picture of laws in the history as in the metaphysics. If this means that the metaphysical picture of scientific realism no longer includes 'fundamental' universal and exceptionless laws, I see no other option than to cut this sense of 'fundamental' loose. It is far from a novel observation that in the history of science, what is conserved through generations of change and revolution is often the "lower-level structure" (Post 1971, 237). I agree with Worrall (1989b) that defeating the PMI is a prior concern for realists than questions about truth or, here, fundamentality (sec. 1). What else should scientific realists take for building a metaphysics than that which has survived centuries of intellectual upheaval? Further discussion of fundamentality, laws of nature, and the current scientific realism debate must be deferred to a future publication.

In my historical discussion, I agree with the general tenor of realist comment on FL: their survival for almost two centuries offers an optimistic rebuttal to the PMI. However, I take exception to two specific historical claims made by Worrall and Psillos. It was not the case that physicists' modern views of the electromagnetic field and (absence of) the aether took hold far earlier than 1905. Second, it was the case that James Clerk Maxwell believed in the existence of a luminiferous aether as the medium for the propagation of light. My central purpose here is not to comment on Psillos's and others' strategy of relying on scientists' historical views to indicate which parts of past scientific theories are suited for realist belief, however, I think this material makes Psillos's strategy difficult to uphold (cf. Cordero 2012). Under Worrall's original historical picture, the experimental basis for applying and testing FL was relatively constant (pace the importance of electro- and magneto-optics). With my periodization, the transition of FL from a material aether theory to modern electrodynamics without a (material) aether overlaps with the development of the quantum theory of solids and of radiation. These developments-culminating with the maser and laser-put new limits on the applicability of FL.

The ceteris paribus view of laws is well suited to the historical trajectory of FL. As the core expression of FL passed through generations of physical experiments and theories, its ceteris paribus clauses changed in content and in number. Following a line of thought from Nagel and Glymour, the specification of ceteris paribus clauses in terms of other theories is an expression of scientific progress. As our understanding of crystals improved, FL's ceteris paribus clauses became better understood and quantitatively related to "solid-state" physics. With high-energy beams of light, qualitatively new phenomena were created, and mathematical expressions-qualitatively different from FL—were devised to describe them. Because of these qualitative differences, there is not a 
well-defined sense in which FL are logically derivable, or mathematically approximate-able, from an underlying quantum theory. This seems to be a general feature of physical theories (Batterman 2012). As Sarkar (2015) recently emphasized, Nagel's theory of inter-theoretic reduction always included qualitative factors. The universal and exceptionless view of laws rejects these qualitative factors because they specify limitations on the applicability of laws of nature, and because they refer to particular physical phenomena and apparatuses. These factors fit naturally with the ceteris paribus view of laws. Diachronically, they link up with Glymour's picture of inter-theoretic relations, and synchronically they form links between physical theories, forming the basis for a unifying view of science.

The ceteris paribus picture of laws with "eliminable" clauses, fits the history of Fresnel's Laws and provides a well-defined framework for understanding scientific structures. The universal exceptionless generalizations picture of laws cannot rebut historicist anti-realism, nor can it ground a contemporary scientific realism; we simply do not have any examples of true universal exceptionless generalizations. Under these conditions, ceteris paribus laws are the better framework for Structural Realism. 


\section{Bibliography}

Batterman, Robert. 2012. "Intertheory Relations in Physics." In The Stanford Encyclopedia of Philosophy, edited by Edward N. Zalta.

Biagioli, Mario. 1996. "From Relativism to Contingentism." In The Disunity of Science: Boundaries, contexts, and power, edited by Peter Galison and David Stump, 189-206. Stanford, CA: Stanford University Press.

Born, Max, and Emil Wolf. 1999. Principles of optics: Electromagnetic theory of propagation, interference and diffraction of light. Seventh (expanded) ed. Cambridge, UK: Cambrdige University Press.

Boyd, Robert W. 2003. Nonlinear Optics. 2nd ed. San Diego, CA: Academic Press.

Bromberg, Joan Lisa. 1991. The Laser in America, 1950-1970. Cambridge, MA: MIT Press.

Brush, Stephen G. 2007. "Predictivism and the periodic table." Studies in History and Philosophy of Science Part A 38 (1): 256-259.

Buchwald, Jed Z. 1985. From Maxwell to Mircophysics: Aspects of Electromagnetic Theory in the Last Quarter of the Nineteenth Century. Chicago: University of Chicago Press.

- 1989. The Rise of the Wave Theory of Light: Optical theory and experiment in the early nineteenth century. Chicago: University of Chicago Press.

1992. "Waves, Philosophers and Historians." PSA: Proceedings of the Biennial Meeting of the Philosophy of Science Association: 205-11.

- 1994. The Creation of Scientific Effects: Heinrich Hertz and electric waves. Chicago: University of Chicago Press.

Cantor, G. N., and M. J. S. Hodge, eds. 1981. Conceptions of Ether: studies in the history of ether theories, 1740-1900. Cambridge, UK: Cambridge University Press.

Cartwright, Nancy. 1980. “The Truth Doesn't Explain Much.” American Philosophical Quarterly 17 (2): 159-163.

-1983. How The Laws of Physics Lie. Oxford: Clarendon Press.

- 1999. The Dappled World. Cambridge: Cambridge University Press.

. 2002. "In Favor of Laws That Are Not Ceteris Paribus after All." Erkenntnis 57 (3): $425-439$. 
Cat, Jordi. 2001. "On Understanding: Maxwell on the Methods of Illustration and Scientific Metaphor." Studies In History and Philosophy of Science Part B: Studies In History and Philosophy of Modern Physics 32 (3): 395-441.

Chakravartty, Anjan. 2007. A Metaphysics for Scientific Realism: Knowing the unobservable. Cambridge, UK: Cambridge University Press.

. 2014. "Scientific Realism." In The Stanford Encyclopedia of Philosophy, Spring 2014 ed., edited by Edward N. Zalta.

Chalmers, Alan. 2001. "Maxwell, Mechanism, and the Nature of Electricity." Physics in Perspective 3 (4): 425-438.

Chang, Hasok. 2003. "Preservative Realism and Its Discontents: Revisiting Caloric." Philosophy of Science 70 (5): 902-912.

Collins, H. M. 1985. Changing order: replication and induction in scientific practice. London: Sage Publications.

Cordero, Alberto. 2012. "Rejected Posits, Realism, and the History of Science." In EPSA Philosophy of Science: Amsterdam 2009, edited by H. de Regt, S. Hartmann, and S. Okasha, 1:23-32. The European Philosophy of Science Association Proceedings. Dordrecht: Springer.

Darrigol, Olivier. 1994. "The Electron Theories of Larmor and Lorentz: A Comparative Study." Historical Studies in the Physical and Biological Sciences 24 (2): 265-336.

-2000. Electrodynamics from Ampere to Einstein. Oxford: Oxford University Press.

Earman, John, and John Roberts. 1999. "“Ceteris Paribus”, There is No Problem of Provisos." Synthese 118 (3): 439-478.

Earman, John, John T. Roberts, and Sheldon Smith. 2002. "Ceteris Paribus Post." Erkenntnis 57 (3): 281-301.

Edwards, Paul N. 2010. A Vast Machine: Computer Models, Climate Data, and the Politics of Global Warming. Cambridge, MA: MIT Press.

Einstein, Albert. (1920) 2007. "Ether and the Theory of Relativity." In The Genesis of General Relativity, edited by Michel Janssen, John D. Norton, Jürgen Renn, Tilman Sauer, and John Stachel, 250:1537-1542. Boston Studies in the Philosophy of Science. Dordrecht: Springer.

1998. Einstein's Miraculous Year: Five papers that changed the face of physics. Edited by John J. Stachel. Princeton, NJ: Princeton University Press. 
Everitt, C. W. F. (1974) 2008. “Maxwell, James Clerk.” In Complete Dictionary of Scientific Biography, 9:198-230. Detroit: Charles Scribner's Sons.

Feyerabend, Paul. 1962. "Explanation, Reduction, and Empiricism." Minnesota Studies in the Philosophy of Science 3 (January): 28-97.

. (1975) 2010. Against Method. New Edition. Introduced by Ian Hacking. London, New York: Verso.

FitzGerald, George Francis. (1888) 1902. "Address to the Mathematical and Physical Section of the British Association." In The Scientific Writings of the Late George Francis FitzGerald, edited by Joseph Larmor, 229-240. London: Longmans, Green, \& co.

French, Steven. 2006. "Sructure as a weapon of the realist." Proceedings of the Aristotelian Society 106 (1): 170-187.

- 2014. The Structure of the World: Metaphysics and Representation. Oxford: Oxford University Press.

Fresnel, Augustin. (1819) 1866. “Appendice.” In Fresnel 1866, 649-653.

- (1823a) 1866. "Extrait d'un Mémoire sur la loi des modifications imprimées a la lumière polarisée par sa réflection totale dans l'interieur des corps transparents." In Fresnel 1866, 753-762.

. (1823b) 1866. "Mémoire sur la loi des modifications que la réflexion imprime a la lumière polarisée.” In Fresnel 1866, 767-799.

1866. Euvres complètes D’Augustin Fresnel. Edited by Henri de Senarmont, Émile Verdet, and Lénor Fresnel. Vol. 1. Paris: Imprimerie Impériale.

Giere, Ronald N. 1999. Science without laws. Chicago: University of Chicago Press.

Glick, Thomas F, ed. 1987. The Comparative reception of relativity. Vol. 103. Boston studies in the philosophy of science. Dordrecht, Holland: D. Reidel.

Glymour, Clark. 1970. “On Some Patterns of Reduction.” Philosophy of Science 37 (3): 340-353.

Godin, Benoit, and Yves Gingras. 2002. "The experimenters' regress: from skepticism to argumentation." Studies In History and Philosophy of Science Part A 33 (1): 133-148.

Grant, Edward. 1981. Much Ado About Nothing: Theories of space and vacuum from the Middle Ages to the scientific revolution. Cambridge, UK: Cambridge University Press.

Hacking, Ian. 1989. "Extragalactic Reality: The Case of Gravitational Lensing." Philosophy of Science 56, no. 4 (December): 555-581. 
Hahn, Th., ed. 2006. International Tables for Crystallography. Vol. A: Space-group symmetry. New York: Wiley.

Hempel, Carl G. 1988. "Provisoes: A Problem concerning the Inferential Function of Scientific Theories." Erkenntnis 28 (2): 147-164.

Hempel, Carl G., and Paul Oppenheim. 1948. "Studies in the Logic of Explanation." Philosophy of Science 15 (2): 135-75.

Hirosige, Tetu. 1969. "Origins of Lorentz' Theory of Electrons and the Concept of the Electromagnetic Field." Historical Studies in the Physical Sciences 1:151-209.

1976. "The Ether Problem, the Mechanistic Worldview, and the Origins of the Theory of Relativity." Historical Studies in the Physical Sciences 7:3-82.

Hoefer, Carl. 2008. "For Fundamentalism." In Nancy Cartwright's philosophy of science, edited by Stephan Hartmann, Carl Hoefer, and Luc Bovens, 307-323. With a reply by Nancy Cartwright. New York: Routledge.

Holton, Gerald James. 1988. Thematic Origins of Scientific Thought: Kepler to Einstein. Rev. ed. Cambridge, MA: Harvard University Press.

Hunt, Bruce J. 1991. The Maxwellians. Ithaca, NY: Cornell University Press.

- 2002. "Electrical Therory and Practice in the Nineteenth Century." In The Modern Physical and Mathematical Sciences, edited by Mary Jo Nye. Cambridge University Press.

. 2014. "Maxwell, Measurement, and the Modes of Electromagnetic Theory." Historical Studies in the Natural Sciences 45 (2): 303-339.

Iggers, Georg G. 2005. Historiography in the Twentieth Century: From scientific objectivity to the postmodern challenge. Translation of Geschichtswissenschaft im 20. Jahrhundert. Middletown, CT: Wesleyan University Press.

Jackson, John David. 1998. Classical Electrodynamics. 3rd ed. New York: Wiley.

Jungnickel, Christa, and Russell McCormmach. 1986. Intellectual mastery of nature: theoretical physics from Ohm to Einstein. Chicago: University of Chicago Press.

Kuhn, Thomas S. (1962) 1996. The Structure of Scientific Revolutions. 3rd ed. Chicago: University of Chicago Press.

. (1970) 1977. "Logic of Discovery or Psychology of Research?” In The Essential Tension: Selected studies of scientific tradition and change, 266-292. Chicago: University of Chicago Press. 
Ladyman, James. 1998. "What is structural realism?" Studies In History and Philosophy of Science Part A 29 (3): 409-424.

. 2009. "Structural Realism." In The Stanford Encyclopedia of Philosophy, edited by Edward N. Zalta.

Ladyman, James, and Don Ross. 2007. Every Thing Must Go: Metaphysics naturalized. With David Spurrett and John G. Collier. Oxford: Oxford University Press.

Landau, L. D., and E. M. Lifshits. 1984. Electrodynamics of Continuous Media. 2nd rev. and enl. ed. Translated by J. B. Sykes, J. S. Bell, and M. J. Kearsley. Vol. 8. Course of Theoretical Physics. Oxford: Pergamon Press.

Lange, Marc. 2000. Natural Laws in Scientific Practice. Oxford: Oxford Univeristy Press.

- 2001. "Review: Scientific Realism: How Science Tracks Truth, by Stathis Psillos." Philosophy of Science 68 (3): 317-320.

Laudan, Larry. 1981. "A Confutation of Convergent Realism.” Philosophy of Science 48, no. 1 (March): 19-49.

Leplin, Jarrett. 2000. "Review: Scientific Realism; How Science Tracks Truth by Stathis Psillos." Mind 109, no. 436 (October): 980-983.

Lorentz, Hendrik A. 1916. The Theory of Electrons and its application to the phenomena of light and radiant heat. 2 nd ed. Leipzig: B. G. Teubner.

Maxwell, James Clerk. 1861a. "On Physical Lines of Force Part II-The Theory of Molecular Vortices applies to Electric Currents.” Philosophical Magazine Series 421 (140-141): 281-291, 338-348.

1861b. "On Physical Lines of Force Part I-The Theory of Molecular Vortices applied to Magnetic Phenomena.” Philosophical Magazine Series 421 (139): 161175.

1865. "A Dynamical Theory of the Electromagnetic Field." Philosophical Transactions of the Royal Society. London 155 (January): 459-512.

. (1878) 1890. “Ether.” In Maxwell 1890, chap. XCVII.

1890. The Scientific Papers of James Clerk Maxwell. Repr. Dover Publications 1965. Edited by W. D. Niven. New York: Cambrdige University Press.

. (1891) 1954. A Treatise on Electricity and Magnetism. Unabridged Third Ed. Edited by W. D. Niven. New York: Dover Publications. 
McCormmach, Russell. 1970. "H. A. Lorentz and the Electromagnetic View of Nature." Isis 61 (4): 459-497.

Michelson, Albert A., and Edward W. Morley. 1887. "On the Relative Motion of the Earth and the Luminiferous Ether." American Journal of Science XXXIV (203): 333-45.

Morrison, Margaret. 1992. "A study in theory unification: The case of Maxwell's electromagnetic theory." Studies in History and Philosophy of Science Part A 23 (1): 103-145.

Mulligan, Joseph F. 2001. “The Aether and Heinrich Hertz's The Principles of Mechanics Presented in a New Form." Physics in Perspective 3 (2): 136-164.

Mumford, Stephen. 2005. "Laws and Lawlessness." Synthese 144 (3): 397-413.

Nagel, Ernest. 1961. The Structure of Science: Problems in the Logic of Scientific Explanation. New York: Harcourt, Brace \& World, Inc.

. 1970. "Issues in the Logic of Reductive Explanations." In Mind, Science, and History, edited by Howard E Kiefer and Milton K. Munitz, 117-137. Albany, NY: State University of New York Press.

Navarro, Jaume, ed. 2018. Ether and Modernity. Forthcoming. Oxford: Oxford Univeristy Press.

Newton, Isaac. (1687) 1999. The Principia: Mathematical principles of natural philosophy. Edited and translated by I. Bernard Cohen and Anne Miller Whitman. Berkeley, CA: University of California Press.

Post, H. R. 1971. "Correspondence, invariance and heuristics: In praise of conservative induction." Studies in History and Philosophy of Science Part A 2 (3): 213-255.

Psillos, Stathis. 1995. "Is Structural Realism the Best of Both Worlds?" Dialectica 49 (1): $15-46$.

1999. Scientific Realism: How Science Tracks Truth. London: Routledge.

2005. "Scientific Realism and Metaphysics." Ratio (new series) XVIII, no. 4 (December): $385-404$.

— 2006. "The Structure, the Whole Structure, and Nothing but the Structure?" Philosophy of Science 73 (5): 560-570.

Putnam, Hilary. 1975. "What Is "Realism"?” Proceedings of the Aristotelian Society 76:177194.

Ruby, Jane E. 1986. "The Origins of Scientific "Law"." Journal of the History of Ideas 47 (3): 341-359. 
Saatsi, Juha. 2005. "Reconsidering the Fresnel-Maxwell theory shift: how the realist can have her cake and EAT it too." Studies in History and Philosophy of Science Part A 36 (3): 509-538.

Sarkar, Sahotra. 2015. "Nagel on reduction." Studies in History and Philosophy of Science 53:43-56.

Schaffner, Kenneth F. 1972. Nineteenth-Century Aether Theories. Oxford: Pergamon Press.

Shapin, Steven, and Simon Schaffer. 1985. Leviathan and the Air-Pump: Hobbes, Boyle, and the experimental life. Princeton, NJ: Princeton University Press.

Siegel, Daniel M. 1981. "Thomson, Maxwell, and the universal ether in Victorian physics." In Cantor and Hodge 1981, 239-268.

- 1991. Innovation in Maxwell's electromagnetic theory: molecular vortices, displacement current, and light. Cambridge, UK: Cambridge University Press.

Staley, Richard. 2008. Einstein's Generation: The Origins of the Relativity Revolution. Chicago: University of Chicago Press.

Stanford, P. Kyle. 2003a. "No Refuge for Realism: Selective Confirmation and the History of Science." Philosophy of Science 70 (5): 913-925.

2003b. "Pyrrhic Victories for Scientific Realism." The Journal of Philosophy 100, no. 11 (November): 553-572.

Stanford, P. Kyle, Paul Humphreys, Katherine Hawley, James Ladyman, and Don Ross. 2010. “Book Symposium: Protecting rainforest realism.” Metascience 19:161-185.

Stanley, Matthew. 2012. "By design: James Clerk Maxwell and the evangelical unification of science." The British Journal for the History of Science 45 (1): 57-73.

- 2015. Huxley's Church and Maxwell's Demon: From theistic science to naturalistic science. Chicago: University of Chicago Press.

Stein, Howard. 1970. "On the Notion of Field in Newton, Maxwell, and Beyond." In Historical and Philosophical Perspective of Science, edited by R. Stuewer, 264-87. Minneapolis: University of Minnesota Press.

. 1989. "Yes, but... Some Skeptical Remarks on Realism and Anti-Realism." Dialectica 43 (1/2): 47-65.

Suppes, Patrick. 2002. Representation and Invariance of Scientific Structures. Stanford: CSLI Publications. 
Warwick, Andrew. 2003. Masters of Theory: Cambridge and the rise of mathematical physics. Chicago: University of Chicago Press.

Wilczek, Frank. 2008. The Lightness of Being: Mass, Ether, and Unification of the Forces. New York: Basic Books.

Worrall, John. 1989a. "Fresnel, Poisson and the white spot: the role of successful predictions in the acceptance of scientific theories." In The Uses of Experiment: Studies in the natural sciences, edited by David Gooding, Trevor Pinch, and Simon Schaffer, 135158. Cambridge, UK: Cambridge University Press.

1989b. "Structural Realism: The Best of Both Worlds?" Dialectica 43 (1-2): 99124.

1994. "How to Remain (Reasonably) Optimistic: Scientific Realism and the "Luminiferous Ether"." PSA: Proceedings of the Biennial Meeting of the Philosophy of Science Association 1:334-342.

Wright, Aaron Sidney. 2016. “A Beautiful Sea: P. A. M. Dirac's epistemology and ontology of the vacuum." Annals of Science 73 (3): 225-256. 